\title{
Auto-Generating Visual Exercises for Learning Program Semantics
}

\author{
Rebecca Smith \\ Rice University \\ Houston, Texas \\ rjs@rice.edu \\ Joe Warren \\ Rice University \\ Houston, Texas \\ jwarren@rice.edu
}

\author{
Terry Tang \\ Rice University \\ Houston, Texas \\ terry.tang@rice.edu \\ Scott Rixner \\ Rice University \\ Houston, Texas \\ rixner@rice.edu
}

\begin{abstract}
Understanding program execution is a challenging task for novice programmers. The semantic rules which determine how execution affects the program state are numerous and complex, and students frequently hold fundamental misconceptions about these rules. If students do not build a correct mental model of program execution early on, they will face substantial hurdles as they try to develop and debug their code. This paper presents VizQuiz, a tool for autogenerating multiple choice quizzes designed to help students gain insight into the semantic rules which govern program execution. VizQuiz provides students with an initial state and a piece of code, and tasks them with mentally tracing the execution of that code and selecting the correct final state. Reference diagrams are used to depict the initial and final states, and as feedback to help students visualize the correct behavior if they select a wrong answer. Feedback is auto-generated, so students can immediately correct their misconceptions and re-attempt.
\end{abstract}

\section{CCS CONCEPTS}

- Social and professional topics $\rightarrow$ Computer science education; $•$ Applied computing $\rightarrow$ Interactive learning environments.

\section{KEYWORDS}

Education; Program semantics; Program mutation

\section{ACM Reference Format:}

Rebecca Smith, Terry Tang, Joe Warren, and Scott Rixner. 2019. AutoGenerating Visual Exercises for Learning Program Semantics. In Innovation and Technology in Computer Science Education (ITiCSE '19), fuly 15-17, 2019, Aberdeen, Scotland UK. ACM, New York, NY, USA, 7 pages. https://doi.org/10.1145/3304221.3319741

Permission to make digital or hard copies of all or part of this work for personal or classroom use is granted without fee provided that copies are not made or distributed for profit or commercial advantage and that copies bear this notice and the full citation on the first page. Copyrights for components of this work owned by others than ACM must be honored. Abstracting with credit is permitted. To copy otherwise, or republish, to post on servers or to redistribute to lists, requires prior specific permission and/or a fee. Request permissions from permissions@acm.org.

ITiCSE '19, fuly 15-17, 2019, Aberdeen, Scotland UK

(C) 2019 Association for Computing Machinery.

ACM ISBN 978-1-4503-6301-3/19/07 . \$ \$15.00

https://doi.org/10.1145/3304221.3319741

\section{INTRODUCTION}

The semantic rules which drive program execution are numerous, complex, and are often not intuitive to novice programmers. Students frequently hold fundamental misconceptions about the effects of program execution on the program state, and these misconceptions create substantial challenges during the development process. Therefore, it is essential to help novice programmers build correct mental models of program execution early on, so that they can effectively develop and debug their programs [2, 14-16, 18, 20].

Much past work has focused on creating tools to help students visualize the program state $[5,7,9,11]$. These visualization tools are valuable aids to students who wish to see the current state of a program. However, such tools typically offer a passive experience. On its own, passive visualization is not enough; the scholarship of teaching and learning provides a wealth of support for the value of learning experiences that actively engage students $[1,3,6,10,13]$. In particular, it links the depth of mental processing that students perform to their ultimate learning and retention [3].

Several past efforts have designed tools for "active code reading" $[8,12,17]$, with the goal of actively engaging students in higher orders of cognition. However, these tools largely lack support for helping students to visualize the program state. Furthermore, most existing tools offer little to no feedback to students on wrong answer selection. UUhistle does combine visualization with interactivity by presenting students with code and asking them to draw reference diagrams from scratch [19]. However, this is a challenging task for beginners, making UUhistle better suited for students that already have preliminary mental models of program execution.

To bridge the gap between the two classes of existing tools in a way that is suitable for beginners, this paper presents VizQuiz. VizQuiz is a multiple choice quiz tool which combines active code reading with program visualization. It provides students with an initial state and a piece of Python code, and asks them to mentally trace the execution of that code. After mentally executing the code, students must select the reference diagram that depicts the correct final state from amongst several distractors. The distractors are not random, nor do they require the instructor to write any code; each is an auto-generated mutation of the correct solution designed to mimic a semantic misconception that students might hold. If a student selects an incorrect state, VizQuiz offers a hint tailored to the specific misconception associated with that distractor. 


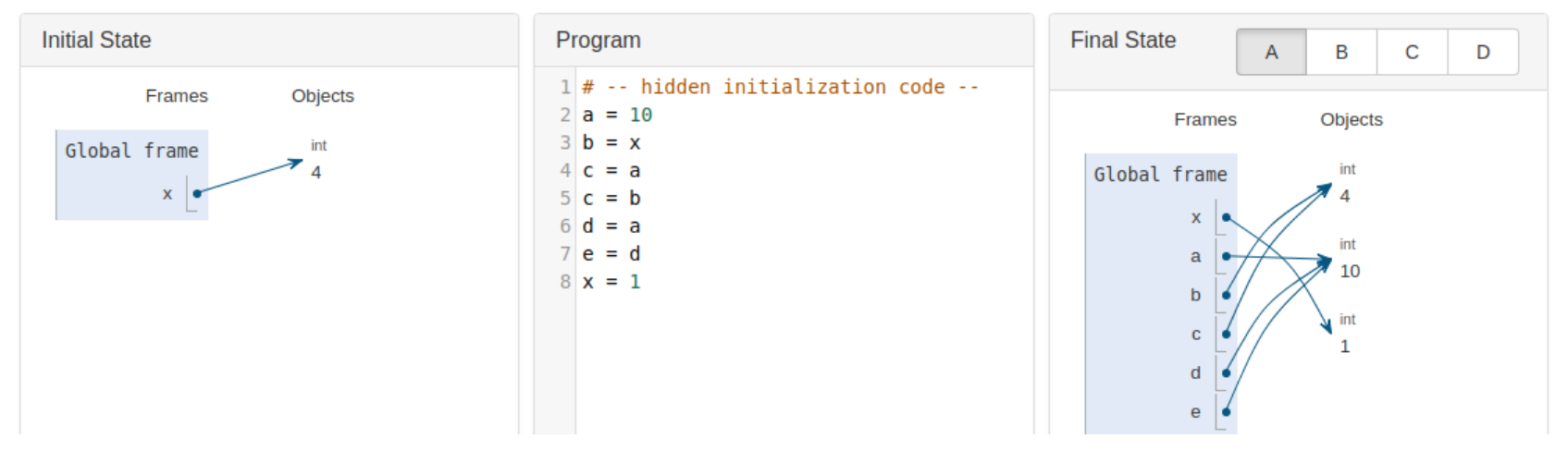

Figure 1: Student Interface

Several factors contribute to the effectiveness of VizQuiz. First, it uniquely provides an interactive visualization experience that is tailored to novice programmers. Second, it scaffolds the learning experience by providing students with several states to choose from and offering automated, targeted feedback. Last, VizQuiz substantially eases the burden on the instructor by automating the tedious process of creating distractors that mimic faulty semantics. Instructors need only specify which misconceptions to target, and VizQuiz will generate the distractors as well as corresponding feedback.

VizQuiz was piloted in a controlled experiment within an introductory class on computational thinking. Students in the control group took three traditional multiple choice quizzes that had been hand-crafted by instructors and refined over several iterations of the course. Students in the experimental group used VizQuiz to take three quizzes that were designed to test the same concepts as the control quizzes. In this study, the experimental group reported average improvements in their computational thinking abilities and critical thinking skills that were greater than the corresponding gains of the control group by a statistically-significant margin, with $p$ values of 0.023 and 0.031 , respectively.

\section{WORKFLOW}

VizQuiz is designed to have an easy and intuitive workflow to support the goals of both students and instructors. Students are required to actively engage with the exercises, but are supported with visualizations and feedback. Instructors are given flexibility in choosing which misconceptions to target, but automation eases the task of generating exercises. Sections 2.1 and 2.2 describe the student and instructor workflows, respectively.

\subsection{Student Workflow}

When using VizQuiz, students are presented with three panes, as shown in Figure 1. The left pane displays a reference diagram representing the initial state of a program, and the middle pane contains the code of a program to be mentally executed on that initial state. The right pane allows the student to toggle between several different reference diagrams, each representing a possible final state. One is the correct final state that would result from applying the given program to the given initial state; the others are distractors designed to target common misconceptions. All
Table 1: Quiz Components

\begin{tabular}{c|cc|cc}
\hline \hline \multirow{2}{*}{ Component } & \multicolumn{2}{|c|}{ Code } & \multicolumn{2}{c|}{ Reference Diagram } \\
\cline { 2 - 6 } & $\begin{array}{c}\text { Auto- } \\
\text { generated? }\end{array}$ & Visible? & $\begin{array}{c}\text { Auto- } \\
\text { generated? }\end{array}$ & Visible? \\
\hline Init. code & No & No & Yes & Yes (left) \\
Applied code & No & Yes (center) & Yes & Yes (right) \\
Mutants & Yes & No & Yes & Yes (right) \\
Hints & Yes & $\begin{array}{c}\text { On wrong } \\
\text { answer }\end{array}$ & Yes & $\begin{array}{c}\text { On wrong } \\
\text { answer }\end{array}$ \\
\hline
\end{tabular}

reference diagrams - for both the initial state and the final states are generated using code adapted from Online Python Tutor [7].

Student must mentally execute the provided code in order to select the correct final state. Upon selecting an incorrect answer, the student is presented with a hint designed to exemplify a concept about which they likely hold a misconception. This hint consists of a small piece of code and a reference diagram illustrating the correct behavior of that code. The student can then correct their misconception and re-attempt the question.

\subsection{Instructor Workflow}

To generate quiz questions, the instructor must first write code for the initial state and the program to be executed. The initialization code is typically brief, serving only to define a few variables. For the fifteen quiz questions used in the evaluation, this code ranged from 1-5 lines. The length of the program to be executed varies according to the desired difficulty and the complexity of the concepts being tested; the programs used in the evaluation ranged from 4-23 lines.

Having written the program to be executed, the instructor can then use VizQuiz's mutation infrastructure to generate mutated variants of that program. A single mutant is created by applying one or more transformations to the program. The mutation framework will be described in greater detail in Section 3.1. Each mutant ultimately corresponds to a single distractor. The mutant code itself is not presented to students; instead, VizQuiz uses these mutants as well as the original program - to generate reference diagrams to be displayed as possible answer choices.

Table 1 summarizes the different components that form a quiz question: initialization code, program to be executed, mutants, and hints. For each component, it indicates whether the code itself 
and/or its corresponding reference diagram is auto-generated or written by the instructor, as well as whether the code and/or its reference diagram are visible to the students. For components that are visible, it specifies the pane in which they appear.

\section{INFRASTRUCTURE}

The infrastructure of VizQuiz can be broken down into two core components. The backend auto-generates distractors and hints based on common student misconceptions. The frontend includes an instructor interface for using the results of auto-generation to build quizzes, as well as a student interface for interacting with these quizzes. While the two components are designed to complement each other, they are de-coupled in order to allow the instructor maximal flexibility. For instance, the instructor might choose to use the distractors or hints generated by the backend within a different context. Or, the instructor could choose to use the frontend framework for visualization and formative assessment, but to modify or even hand-write from scratch one or more distractors or hints.

\subsection{Backend: Distractor \& Hint Generation}

Crafting mutants that capture semantic misconceptions is not a simple task; it often requires sophisticated, multi-line transformations. Hand-writing high quality mutants would be an onerous burden for the instructor. Therefore, VizQuiz auto-generates mutants using a modified version of mutpy [4]. mutpy is a mutation-testing tool for Python which takes an input program and randomly applies simple transformations to that program in order to generate mutants. The built-in transformations are very basic and typically affect only a single line; for instance, changing a plus sign into a minus sign, or changing the value of a constant. Such transformations are effective for mutpy's original purpose of mutation testing. However, they do not reflect realistic misconceptions that students hold, and therefore are ill-suited to an educational context.

To make mutpy generate code that mimics semantic misconceptions, three key changes were made. First, the tool was modified to consistently apply the same transformation throughout the input program, rather than randomly selecting a single line to mutate. Second, thirty-two new, complex transformations were implemented.

\section{Table 2: Selection of Transformations}

\begin{tabular}{c|l}
\hline \hline Transformation & Student erroneously believes that... \\
\hline AndOrHigherThanNot & $\begin{array}{l}\text { and/or have higher precedence than not } \\
\text { Augmented assignment operations } \\
\text { (e.g., +=) mutate the lefthand side } \\
\text { References in Python can point to } \\
\text { other references (rather than objects) } \\
\text { Functions don't have side effects, thus } \\
\text { calls whose return values are unused can } \\
\text { be ignored } \\
\text { ChainsReferences } \\
\text { DeleteUnusedCallion involving two ints returns a float } \\
\text { if and elif are not mutually exclusive } \\
\text { DivisFractional } \\
\text { ElifIsIf } \\
\text { EvaluateLeftic expressions are evaluated } \\
\text { left-to-right (ignoring order of operations) } \\
\text { print is equivalent to return } \\
\text { ScopeDoesNoturn }\end{array}$ \\
\hline
\end{tabular}

\begin{tabular}{c|l|l}
\hline \hline Input & Mutant & Hint \\
\hline $\mathrm{a}=10$ & $\mathrm{a}=10$ & $\mathrm{q}=5$ \\
$\mathrm{~b}=\mathrm{x}$ & $\mathrm{b}=\mathrm{x}$ & $\mathrm{r}=\mathrm{q}$ \\
$\mathrm{c}=\mathrm{a}$ & $\mathrm{c}=\mathrm{a}$ & $\mathrm{q}=10$ \\
$\mathrm{c}=\mathrm{b}$ & $\mathrm{c}=\mathrm{b}$ & \\
$\mathrm{d}=\mathrm{a}$ & $\mathrm{d}=\mathrm{a}$ & \\
$\mathrm{e}=\mathrm{d}$ & $\mathrm{e}=\mathrm{d}$ & \\
$\mathrm{x}=1$ & $\mathrm{x}=1$ & \\
& $\mathrm{c}=\mathrm{x}$ & \\
& $\mathrm{b}=\mathrm{x}$ & \\
\hline
\end{tabular}

Figure 2: Auto-Generation Input \& Outputs

Each of these was designed to target a specific misconception that is common amongst novice programmers. Effectively, these transformations serve to mimic faulty semantics. If the student holds the misconception being targeted by a given mutant, the corresponding reference diagram will reflect what they erroneously believe would be the program state after executing the input program.

The complete collection of transformations is too numerous to list here. Instead, Table 2 presents a selection of transformations as examples. These cover misconceptions on a range of topics, which include, but are not limited to, mutation (ChainsReferences, AugAssignsMutate) scope (ScopeDoesNotExist), order of operations (EvaluateLeftToRight, AndOrHigherThanNot) and control flow (PrintIsReturn, ElifIsIf). While the existing thirty-two transformations offer a rich variety of options to instructors, the framework is designed to be extensible, such that new transformations could easily be added to suit additional use cases.

The third change was to tag each transformation with a hint. When the instructor selects a transformation to be used to generate a distractor, the associated hint is output along with the mutant program. This hint consists of a short snippet of code that is a minimal working example of the concept underlying the misconception.

Figure 2 shows a sample input and the associated output. In this example, the instructor has selected the ChainsReferences misconception, which targets the case where a student erroneously believes that Python references can point to other references. Under this misconception, the student would expect re-assignment of a single variable to change the values of all aliases of that variable. Thus, the mutant code explicitly re-assigns each of these aliases to mimic this misconception. The student will not see this mutant code, but will be presented with its corresponding reference diagram. The hint involves a single re-assignment, and would be presented alongside its reference diagram to illustrate that the alias, $r$, actually remains unchanged when $q$ is re-assigned. From this, the student can try to identify and correct the misconception.

\subsection{Frontend: Quiz Framework}

After generating each (mutant, hint) pair, the instructor is ready to use VizQuiz's instructor interface to create a new quiz. For each question, the instructor simply inputs all of the code: the initialization code, the program to be executed, the mutants and the hints. VizQuiz takes care of rendering the corresponding reference diagrams for the initial state and potential final states. 
Table 3: Average Survey Responses, Spring 2018 (4-point Likert Scale)

\begin{tabular}{|c|c|c|c|c|c|c|c|c|c|c|}
\hline \multirow{2}{*}{\multicolumn{2}{|c|}{ Question }} & \multicolumn{4}{|c|}{ Control Group } & \multicolumn{4}{|c|}{ Experimental Group } & \multirow{2}{*}{ p-value } \\
\hline & & $\mathrm{n}$ & Pre-survey & Post-survey & Delta & $\mathrm{n}$ & Pre-survey & Post-survey & Delta & \\
\hline $1 \mathrm{~A}$ & $\begin{array}{l}\text { Please characterize your current level } \\
\text { of understanding concerning how } \\
\text { executing code affects the program state. }\end{array}$ & 29 & 1.897 & 2.759 & +0.862 & 28 & 1.607 & 2.929 & +1.321 & 0.013 \\
\hline 1B & $\begin{array}{l}\text { How confident are you that you will } \\
\text { successfully complete this course? }\end{array}$ & 29 & 2.931 & 2.966 & +0.034 & 28 & 2.537 & 2.893 & +0.357 & 0.034 \\
\hline $1 \mathrm{C}$ & $\begin{array}{l}\text { How helpful were quizzes } 1-3 \text { in } \\
\text { improving your understanding of how } \\
\text { simple Python programs are executed? }\end{array}$ & 29 & N/A & 2.690 & N/A & 28 & $\mathrm{~N} / \mathrm{A}$ & 2.679 & N/A & 0.477 \\
\hline
\end{tabular}

Recall from Table 1 that the correct final state is the reference diagram generated from the instructor-written program, while the distractors are the reference diagrams of the mutants. If the student selects the correct final state, they proceed to the next question; otherwise, they receive the hint corresponding to the specific distractor they chose. As mentioned in Section 3.1, hints are presented as both a snippet of code and its the corresponding reference diagram, allowing students to visualize the correct behavior of the operation(s) they misunderstood.

Since VizQuiz is designed to be a formative assessment, students can make multiple attempts with no penalty. However, elements of randomness ensure that students practice mentally executing code rather than mindlessly, exhaustively trying each answer choice. Not only is the order of the answer choices shuffled between attempts, but the questions themselves may actually change slightly. Before each attempt, VizQuiz re-executes the initialization code to generate the initial state. The instructor can design this code such that the initial variables' values are randomized, resulting in a fresh challenge for the students on each subsequent attempt.

\section{METHODOLOGY}

VizQuiz was evaluated within an introductory course on computational thinking. This course has no prerequisites, is taken by a mix of majors and non-majors, and is offered during both semesters. The fall version is typically much larger, with multiple sections and a high proportion of intended majors. The spring version is a single section, and tends to include a greater proportion of non-majors.

A preliminary study ran in the spring of 2018 , and a more indepth follow-up study ran in the fall of 2018. At the start of each semester, students took a pre-survey which asked them to rate their confidence on a Likert scale, and also asked for a small amount of demographic information including their level of prior experience.

The study then ran during the first two weeks of the semester. The course utilizes a flipped classroom model in which students watch several brief video lectures before each class and take a multiple choice quiz on the information presented in those videos. For the purposes of this study, students were divided into a control group and an experimental group. Their group assignment determined the format they used for the first three pre-class quizzes. All students watched the same videos, and thus each pair of quizzes was designed to target the same high-level concepts: expressions, functions, and conditionals. However, the experimental group took three auto-generated quizzes within the VizQuiz framework, while the control group took three "traditional" multiple choice quizzes, composed of entirely different questions: textual questions that had been hand-crafted and refined by instructors during previous iterations of the course. All quizzes consisted of five questions.

In the initial spring study, students were randomly assigned to treatment groups. However, analyzing the results revealed that, by chance, the control group had notably higher prior experience and initial confidence than the experimental group. To avoid this issue in the fall, students were first sub-divided based on their experience level, as reported in the pre-survey. Then, each sub-group was divided randomly and evenly amongst the two treatments.

In both semesters, all students were asked to complete a postsurvey. In the spring, the post-survey mirrored the confidence questions from the pre-survey. In the fall, students were also asked to rate their agreement with a series of statements regarding the quizzes. Additionally, the experimental group was given the opportunity to provide open-ended qualitative feedback on VizQuiz.

\section{EVALUATION}

As outlined in Section 4, both iterations of the study collected quantitative feedback using a Likert scale. In the spring semester, a 4-point Likert scale was used; in fall semester this was expanded to a 5-point scale to allow students to respond at a finer granularity. Sections 5.1 and 5.2 discuss the quantitative feedback from the spring and fall semesters, respectively. Section 5.3 presents selections from the qualitative feedback from the fall semester.

\subsection{Quantitative Feedback, Spring 2018}

Table 3 summarizes the quantitative results from the spring semester. This table is divided into four sections. The left section lists the questions that were asked, labeled $(1 \mathrm{~A}, 1 \mathrm{~B}, 1 \mathrm{C})$ for ease of reference. The two middle sections summarize the responses of the control and experimental groups, with sub-columns for sample size ("n"), average pre-survey response ("Pre-survey"), average postsurvey response ("Post-survey"), and average intra-group change between surveys ("Delta"). The third question was only asked on the post-survey, since it pertained to the students' experience with the quizzes. Thus, its pre-survey and delta values are listed as N/A.

As stated in Section 4, despite the use of random assignment, there was a disparity in the two groups' initial confidence levels. Specifically, the control group had notably higher initial confidence. However, while the experimental group began with less confidence, they reported significantly higher deltas after using VizQuiz, and 
Table 4: Average Self-Reported Confidence, Fall 2018 (5-point Likert Scale)

\begin{tabular}{|c|c|c|c|c|c|c|c|c|c|c|}
\hline \multirow{2}{*}{\multicolumn{2}{|c|}{ "Please rate your confidence in the following:" }} & \multicolumn{4}{|c|}{ Control Group } & \multicolumn{4}{|c|}{ Experimental Group } & \multirow{2}{*}{ p-value } \\
\hline & & $\mathrm{n}$ & Pre-survey & Post-survey & Delta & $\mathrm{n}$ & Pre-survey & Post-survey & Delta & \\
\hline $2 \mathrm{~A}$ & $\begin{array}{l}\text { Your understanding of how executing } \\
\text { code affects the program state } \\
\text { (including variables and their values) }\end{array}$ & 86 & 3.212 & 3.882 & +0.670 & 104 & 2.942 & 3.779 & +0.837 & 0.149 \\
\hline $2 \mathrm{~B}$ & Your ability to complete the class & 87 & 4.314 & 4.291 & -0.023 & 104 & 4.106 & 4.260 & +0.154 & 0.072 \\
\hline $2 \mathrm{C}$ & Your computational thinking abilities & 54 & 3.679 & 3.660 & -0.019 & 77 & 3.260 & 3.584 & +0.324 & 0.023 \\
\hline
\end{tabular}

Table 5: Average Agreement, Fall 2018 (5-point Likert Scale)

\begin{tabular}{ll|cc|cc|c}
\hline \hline \multirow{2}{*}{ "Please rate your agreement with each of the following statements:" } & \multicolumn{2}{|c|}{ Control Group } & \multicolumn{2}{|c|}{ Experimental Group } & \multirow{2}{*}{ p-value } \\
\cline { 3 - 5 } & $\mathrm{n}$ & Post-survey & $\mathrm{n}$ & Post-survey & \\
\hline 3A & Quizzes 1-3 improved my understanding of the program state & 88 & 4.00 & 104 & 4.16 & 0.058 \\
3B & Quizzes 1-3 improved my ability to reason about code execution & 88 & 3.99 & 105 & 4.14 & 0.067 \\
3C & Quizzes 1-3 improved my critical thinking skills & 88 & 3.77 & 105 & 4.01 & $\mathbf{0 . 0 3 1}$ \\
3D & Quizzes 1-3 helped me learn to read simple Python programs & 88 & 4.17 & 105 & 4.36 & $\mathbf{0 . 0 3 2}$ \\
3E & Quizzes 1-3 helped me learn to think like a computer scientist & 88 & 3.86 & 105 & 4.06 & 0.053 \\
\hline
\end{tabular}

Figure 3: Agreement Distribution, Fall 2018 (5-point Likert Scale)

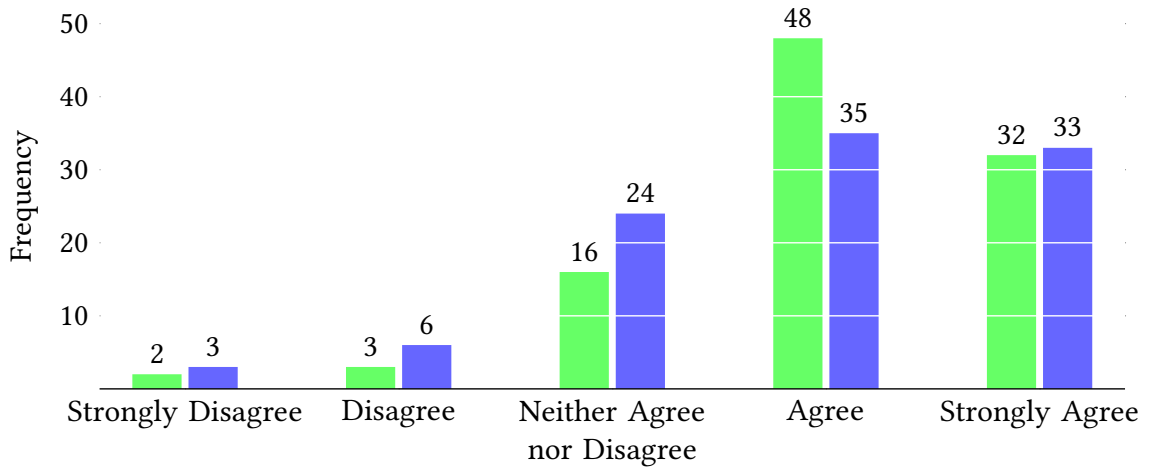

VizQuiz helped me to...

(4A) Visualize code execution and its affects on the program state

(4B) Identify and fix my misconceptions about Python by the end of the experiment they had nearly closed the gap (as in 1B) or even surpassed the control group (as in 1A).

The rightmost column shows the results of using a one-tailed unpaired t-test to compare the two groups. For questions $1 \mathrm{~A}$ and $1 \mathrm{~B}$, which appeared on both the pre- and post-surveys, significance testing was performed on the deltas. For question 1C, which only appeared on the post-survey, significance testing was performed directly on the responses to the post-survey.

The p-values for questions $1 \mathrm{~A}$ and $1 \mathrm{~B}$ were 0.013 and 0.034 , respectively, indicating that the improvements in the experimental group's confidence were greater than those of the control group to a statistically significant degree. These results indicate that VizQuiz likely had a positive impact not only on students' confidence in the specific skills that it targeted - understanding the effects of execution on the program state $(1 \mathrm{~A})-$, but also on their overall confidence in their ability to complete the course (1B).

\subsection{Quantitative Feedback, Fall 2018}

Tables 4 and 5 present quantitative results from the fall semester. The questions in Table 4 asked students to self-report confidence, and appeared on both surveys; those in Table 5 asked students to report agreement with statements regarding the quizzes, and appeared only on the post-survey. Results are again broken down by treatment group, with the rightmost column showing the p-value from a one-tailed unpaired t-test comparing the two groups.

Interestingly, the results for questions $2 \mathrm{~A}$ and $2 \mathrm{~B}-$ which were nearly identical to $1 \mathrm{~A}$ and $1 \mathrm{~B}$ from the spring - were not statistically significant at the $p=0.05$ level in this iteration of the study. However, the responses to question $2 \mathrm{C}-$ which asked students about their confidence in their computational thinking skills - yielded a pvalue of 0.023 , which is considered statistically significant.

Table 5 includes two more statistically significant results at the $p=0.05$ level. First, students that interacted with VizQuiz found their experience to be significantly more effective in improving their critical thinking skills as compared to the control group ( $p=0.031)$. Second, it appears that VizQuiz may offer auxiliary benefits, as students in the experimental group also rated their experience as being significantly more effective in helping them learn to read simple Python programs $(p=0.032)$.

Further, students in the experimental group were asked to rate their agreement with two statements regarding the effectiveness 
Table 6: Selected Feedback, Fall 2018 (Constructive)

\begin{tabular}{l}
\hline \hline \multicolumn{1}{c}{ What was the worst part of using VizQuiz? } \\
Sometimes the arrows were a little confusing, but it wasn't a \\
huge issue. \\
When you couldn't understand the hint it was difficult to figure \\
out what your mistake was. \\
At first I was confused about what we were supposed to do and \\
what it was asking of me but I got the hang of it pretty quickly. \\
It was kind of boring because you couldn't write any actual \\
code or edit anything. \\
It was annoying how it would change questions after a wrong \\
answer, but it makes since why it did this. \\
It very time consuming. \\
N/A. I love VizQuiz! That love doesn't stem from easy material \\
or a less strenuous testing format; I just think that it is a great \\
way to learn.
\end{tabular}

Table 7: Selected Feedback, Fall 2018 (Positive)

What was the best part of using VizQuiz?

It's a good tool for furthering understanding and training you to follow and execute the code.

If I submitted an incorrect answer, [VizQuiz] would provide me with an example of correct usage of some code I misinterpreted. I could then try again and focus on correcting my mistake. Gain a deeper understanding of the method in which code is executed. Also there are multiple attempts so if you ever don't understand something you get another chance to understand. It was clear and easy to understand. The problems were not extremely complex and could be easily done on paper. The hints given when you made a mistake allowed you to correct your understanding of how coding should work. It was a great way to understand Python in a conceptual and fine-tuned way that made sure I didn't skip over crucial details about Python syntax and logic.

[VizQuiz] really helped me visualize the sequence of code and how it is like path of instructions. Since I came into the class with zero coding experience, [VizQuiz] was a nice way to visualize code without actually doing it.

VizQuiz. The average responses were 4.01 (4A) and 3.88 (4B); Figure 3 shows a full breakdown of the results. These results indicate that students largely found VizQuiz to be a valuable aid for visualizing program execution and correcting semantic misconceptions.

\subsection{Qualitative Feedback, Fall 2018}

Last, the post-survey in the fall semester solicited qualitative feedback from the group that interacted with VizQuiz. Students were asked to provide balanced feedback - specifically, they were asked to report both the worst and best parts of their experience with VizQuiz. Tables 6 and 7 contain a selection of responses to these two questions. The constructive criticism, in Table 6, noted that VizQuiz could be challenging and perhaps a bit confusing initially due to the unfamiliar format; however, students noted that they "got the hang of it pretty quickly", and recognized the value of certain design decisions that added difficulty (e.g., questions changing between attempts). On the other hand, the positive comments underscored the value of VizQuiz in helping students to gain a deeper understanding of Python semantics through the combination of mental code execution and visualization. Many comments praised specific features of the tool, such as the visual aids, the hints, and the ability to re-attempt questions until their understanding was solidified.

\section{CONCLUSION}

Building correct mental models of program semantics is critical to students' success in developing and debugging programs. If semantic misconceptions are not corrected early on, they can have cascading effects as students attempt to layer increasingly complex concepts on top of a faulty foundation. Therefore, it is essential to support the development of correct mental models of program semantics from the start. To that end, this paper has presented VizQuiz, a framework for auto-generating multiple choice quizzes to help introductory students build mental models of program execution.

For students, VizQuiz was designed to provide a uniquely interactive visualization experience. It engages them in active code reading, and provides visual feedback to aid them in correcting any misconceptions they hold. Because this feedback is automated, students receive it instantaneously, so that they can immediately work to identify and correct their misconceptions. Multiple attempts create a low-stakes environment well-suited to beginners, allowing students to engage in intensive practice until they achieve mastery.

For instructors, VizQuiz was designed to balance flexibility with automation. It gives the instructor the freedom to choose which specific misconceptions they'd like to target, but automates the tedious work of crafting distractors that accurately reflect those misconceptions. Along with each distractor, it outputs a hint designed to help students correct the underlying misconception. Currently, VizQuiz supports over thirty transformations, corresponding to misconceptions on a wide range of topics such as mutation, scope, and control flow. Further, it is designed to be extensible, and additional transformations could easily be added.

VizQuiz was evaluated in a controlled experiment within an introductory course on computational thinking, where it was compared alongside a more traditional quiz model. In this study, students that interacted with VizQuiz reported significantly greater improvements in their computational thinking abilities and critical thinking skills as compared to the control group, with p-values of 0.023 and 0.031 , respectively. Further, qualitative feedback highlighted the effectiveness of VizQuiz in training students to mentally execute code and helping them to correct their underlying misconceptions.

Helping students gain a firm grasp of the complex semantic rules that govern program execution is an important and challenging task. By providing instructors with an easy means of generating exercises that couple targeted practice with formative feedback, VizQuiz substantially eases this task.

\section{ACKNOWLEDGEMENTS}

This work is supported in part by NSF Award CNS-1619124. 


\section{REFERENCES}

[1] Charles C. Bonwell and James A. Eison. 1991. Active Learning: Creating Excitement in the Classroom. ERIC.

[2] Matt Bower and Katrina Falkner. [n. d.]. Computational Thinking, the Notional Machine, Pre-service Teachers, and Research Opportunities.

[3] Fergus I.M. Craik and Robert S. Lockhart. 1972. Levels of Processing: A Framework for Memory Research. Journal of Verbal Learning and Verbal Behavior 11, 6 (1972), 671-684.

[4] Anna Derezinska and Konrad Halas. 2014. Experimental Evaluation of Mutation Testing Approaches to Python Programs. In Proceedings of the 7th IEEE International Conference on Software Testing, Verification, and Validation Workshops (ICST'14).

[5] Stephan Diehl. 2007. Software Visualization: Visualizing the Structure, Behaviour, and Evolution of Software. Springer Science \& Business Media.

[6] Scott Freeman, Sarah L. Eddy, Miles McDonough, Michelle K. Smith, Nnadozie Okoroafor, Hannah Jordt, and Mary P. Wenderoth. 2014. Active Learning Increases Student Performance in Science, Engineering, and Mathematics. Proceedings of the National Academy of Sciences 111, 23 (2014).

[7] Philip J. Guo. 2013. Online Python Tutor: Embeddable Web-Based Program Visualization for CS Education. In Proceedings of the 44th ACM Technical Symposium on Computer Science Education (SIGCSE '13). Denver, CO.

[8] Daniel M. Hoffman, Ming Lu, and Tim Pelton. 2011. A Web-Based Generation and Delivery System for Active Code Reading. In Proceedings of the 42nd ACM Technical Symposium on Computer Science Education (SIGCSE '11). Dallas, TX

[9] Jhilmil Jain, James H. Cross II, T. Dean Hendrix, and Larry A. Barowski. [n. d.]. Experimental Evaluation of Animated-Verifying Object Viewers for Java. In Proceedings of the 3rd ACM Symposium on Software Visualization (SoftVis '06).
[10] David W. Johnson, Roger T. Johnson, and Karl A Smith. 1998. Active Learning: Cooperation in the College Classroom. ERIC.

[11] Michael Kölling, Bruce Quig, Andrew Patterson, and John Rosenberg. 2003. The BlueJ System and its Pedagogy. Computer Science Education 13, 4 (2003).

[12] Niko Myller. 2007. Automatic Generation of Prediction Questions During Program Visualization. Electronic Notes in Theoretical Computer Science 178 (2007).

[13] Thomas L. Naps, Guido Rößling, Vicki Almstrum, Wanda Dann, Rudolf Fleischer, Chris Hundhausen, Ari Korhonen, Lauri Malmi, Myles McNally, and Susan Rodger. 2002. Exploring the Role of Visualization and Engagement in Computer Science Education. In ACM SIGCSE Bulletin, Vol. 35. ACM, 131-152.

[14] Donald A. Norman. 2014. Some Observations on Mental Models. In Mental Models. Psychology Press, 15-22.

[15] Andrew Ortony and David E. Rumelhart. 2017. The Representation of Knowledge in Memory. In Schooling and the Acquisition of Knowledge. Routledge, 99-135.

[16] Vennila Ramalingam, Deborah LaBelle, and Susan Wiedenbeck. 2004. Self-Efficacy and Mental Models in Learning to Program. In Proceedings of the 9th ACM International Conference on Innovation and Technology in Computer Science Education (ITiCSE '04). Leeds, UK.

[17] Takayuki Sekiya and Kazunori Yamaguchi. 2013. Tracing Quiz Set to Identify Novices' Programming Misconceptions. In Proceedings of the 13th Koli Calling International Conference on Computing Education Research. Koli, Finland.

[18] Juha Sorva. 2013. Notional Machines and Introductory Programming Education. ACM Transactions on Computing Education 13, 2 (2013).

[19] Juha Sorva and Teemu Sirkiä. 2010. UUhistle: A Software Tool for Visual Program Simulation. In Proceedings of the 10th Koli Calling International Conference on Computing Education Research. Koli, Finland.

[20] Lynn Westbrook. 2006. Mental Models: A Theoretical Overview and Preliminary Study. Fournal of Information Science 32, 6 (2006), 563-579. 